\title{
Material slow light does not enhance Beer-Lambert absorption
}

\author{
S. Chin, I. Dicaire, J. C. Beugnot, S. F. Mafang, M. G. Herraez* and Luc Thévenaz \\ Ecole Polytechnique Fédérale de Lausanne, STI-GR-SCI Station 11, CH-1015 Lausanne, Switzerland \\ * Department of Electronics, University of Alcalá de Henares, E-28805 Madrid, Spain \\ sanghoon.chin@epfl.ch, luc.thevenaz@epfl.ch and miguelg@depeca.uah.es
}

\begin{abstract}
We experimentally demonstrate that material slow light does not enhance Beer-Lambert absorption. A $26 \%$ group velocity reduction induced by stimulated Brillouin scattering in a gasfilled microstructured fiber caused no observable change in the measured absorption.

(C)2009 Optical Society of America

OCIS codes: (060.4370) Nonlinear optics, fibers; (290.5900) Scattering, stimulated Brillouin; (350.5500) Propagation; (060.2370) Fiber optics sensors; (060.5295) Photonic crystal fibers
\end{abstract}

\section{Introduction}

Slow and fast light systems are special optical media in which the propagation is strongly altered to produce very slow (or fast) group velocities. Slow \& fast light has attracted considerable interest in the photonic community due to its large potential applications. The main target application of slow light so far has been the achievement of alloptical delay lines, all-optical buffers and signal synchronization elements for future all-optical high capacity networks [1]. These target applications benefit from the group delay changes caused by the slow light element as an indirect consequence of the modification of the group velocity of the medium. However, the most remarkable feature of slow light media which is the possibility to achieve extreme changes in the group velocity remains unexploited. Recently, some research effort has been devoted to understanding the possibilities of slow light in enhancing light-matter interactions. For instance, the role of slow light in enhancing nonlinear effects has been theoretically investigated [2,3]. This enhancement is due to two contributions: the longer transient time of light pulses in optical media and the higher energy density due to spatial pulse compression. It has also been theoretically and experimentally proved that the extreme dispersion of slow light can lead to an enhancement of the spectral sensitivity of interferometers [4,5]. The role of slow light in gyroscopes has also been theoretically investigated [6]. Recent works $[7,8]$ have theoretically demonstrated that Beer-Lambert-Bouguer (BLB) absorption can be increased while light transmits through a special kind of microstructured photonic crystal (PC) cuvette. This absorption enhancement was attributed in $[7,8]$ to the slow group velocity in the cuvette. A simpler interpretation of these results would be that the PC structure acts as a quasi-cavity. The light bounces back and forth in the quasi-cavity several times, resulting in a longer interaction length with the liquid analyte. The experimental proof of these results seems very challenging considering the dimensions of the microstructure and the degree of infiltration required.

In this paper, we show that material slow light (slow light in travelling-wave media) has no impact on BeerLambert absorption. This demonstration is done experimentally, using a special kind of gas cell. A photonic crystal fiber (PCF) with solid core plays the role of the gas cell. The fiber holes are filled with gaseous acetylene at reduced pressure. A small fraction of the mode field (the evanescent field) propagates through the holes and thus experiences Beer-Lambert absorption. The fiber is made of a solid silica core, so that it can also be used as a Brillouin slow light medium. The absorption can therefore be measured under normal and slow-light conditions without modifying the experimental implementation. By comparing the two regimes, we can observe if the slow light has any impact on the absorption by the gas. The results indicate that Beer-Lambert absorption is not at all affected by slow light. To our knowledge, this is the first experimental observation of the effect of material slow light on light-matter interaction.

\section{Principle}

The absorption of a gas analyte is usually expressed by means of the Beer-Lambert (BL) law. If light with an intensity $I_{\mathrm{o}}$ propagates through the gas cell, the transmitted intensity decays exponentially as $I=I_{\mathrm{o}} \exp [-\alpha \cdot \mathrm{C} \cdot L]$, where $C$ is the gas concentration, $L$ is optical path length and $\alpha$ is the molar absorption coefficient, which is a unique property of the gas, related to the imaginary part of the refractive index. Previous papers (ref [7], eq. 3) have suggested that the absorption coefficient is inversely proportional to the group velocity. In this paper we show experimentally that $\alpha$ is not related to the group velocity. To clarify the relationship between BL absorption and slow light, a solid-core microstructured optical fiber replaces the classical gas cell. There are two main reasons for this: first, in such fiber, the optical mode is mainly confined to the solid core. Thus, the velocity of light propagating through the PCF can be controlled by manipulating the material properties of the core using a narrowband gain 


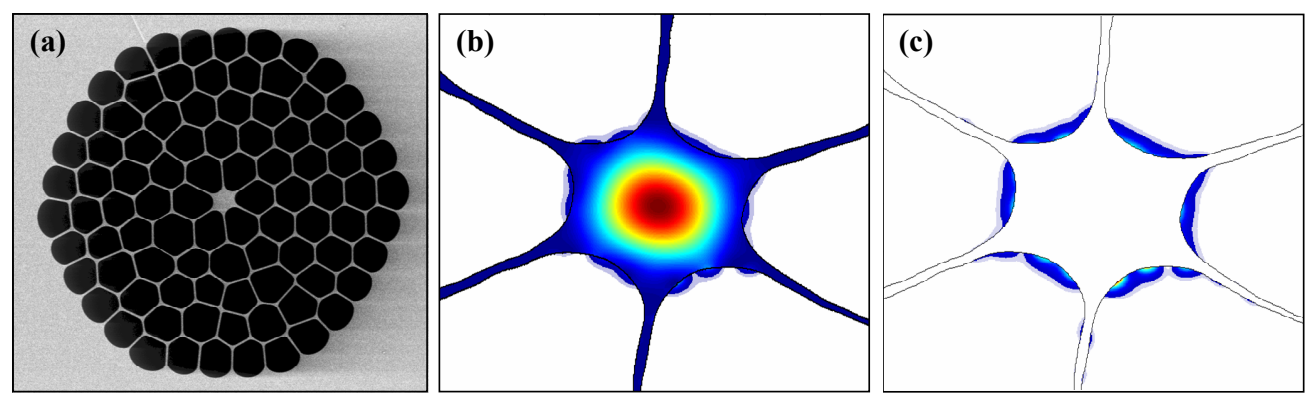

Figure.1: (a) SEM image of the solid-core microstructured fiber. (b) Calculated mode field distribution of the fundamental mode and (c) the small evanescent fraction of the guided field present in air holes.

process such as stimulated Brillouin scattering. Second, with a proper choice of the fiber geometry, a non-negligible fraction of the guided light propagates through the holes filled with gas, and hence can probe Beer-Lambert absorption. The key advantage of this setup is that the effects of slow light and absorption are totally de-coupled, and hence we can vary one without having a direct impact on the other. Under these conditions, the equation of BLB absorption can be slightly modified as

$$
I=I_{o} \exp [-N \cdot \sigma \cdot f \cdot L]
$$

where $N$ is the gas molecular density, $\sigma$ is the absorption cross section, $f$ is the fraction of light in holes and $L$ is the optical length. Figure 1a shows the SEM image of the single-mode PCF filled with acetylene in the holes. Using the source-model technique (SMT) [9], we calculated the mode field distribution along the core cross section of the PCF at a wavelength of $1535 \mathrm{~nm}$, as shown in Figure 1b. The evanescent part of the guided field propagating in the gas, representing $2.9 \%$ of the total power, is shown in Figure 1c.

\section{Experimental results}

As a Brillouin gain medium, a $9.18 \mathrm{~m}$-long PCF was used. The Brillouin spectrum of this fiber was characterized, showing a Brillouin shift of $10.85 \mathrm{GHz}$ and an SBS gain bandwidth of $38 \mathrm{MHz}$. A commercial distributed feedback (DFB) laser diode, operating at $1535 \mathrm{~nm}$, was used as the light source and its output was split using a directional coupler. One branch was strongly boosted using a high power erbium-doped fiber amplifier (EDFA) with $30 \mathrm{dBm}$ saturation power so as to play the role of the Brillouin pump. The output of the EDFA was precisely controlled by a variable optical attenuator before entering into the gas cell. The other branch was modulated through an electro-optic Mach-Zehnder intensity modulator (EOM) at the Brillouin frequency shift so as to generate two first-order sidebands in a suppressed carrier configuration. The lower-frequency sideband was then filtered by a fiber Bragg grating (FBG) and launched into the fiber as the signal frequency.

We first measured the amount of group velocity change as a function of the pump power. For this measurement, the frequencies of the signal and pump waves were spectrally placed in a region where absorption resonances are absent. To determine precisely the SBS-induced group delay, the signal wave was sinusoidally modulated at $1 \mathrm{MHz}$ by another external EOM. The phase of the sine wave after propagation through the PCF was measured while the pump power was incremented by $100 \mathrm{~mW}$ steps from 0 to $600 \mathrm{~mW}$. This way the group delay change achieved through the PCF for different pump powers was accurately determined from the differential phase shift. Additionally, the transit time through the fiber was also determined by making the same measurement with and without the PCF. The group velocity change is quantified by defining a slow-down factor $S$ corresponding to the ratio between the transit times through the gas cell with and without the pump, respectively. The largest time delay achieved through the PCF was $11.7 \mathrm{~ns}$, corresponding to $26 \%$ reduction of the group velocity, at a pump power of $600 \mathrm{~mW}$.

To observe an absorption resonance of acetylene, the frequency of the signal was swept all over the absorption line, simply by introducing a slow variation of the current applied to the initial DFB laser. Since the signal and pump waves were generated from the same DFB laser source, the pump frequency was also swept perfectly synchronously, preserving the exact spectral distance between signal and pump matching the Brillouin shift. Consequently, the pump constantly generated a Brillouin gain resonance centered at the signal frequency, even though the signal frequency was swept across the absorption line. The intensity of the signal wave was also sinusoidally modulated at $100 \mathrm{kHz}$ by an EOM to obtain a clean signal using a lock-in detection. The signal wave was then amplified using another EDFA, so as to even further improve the signal-to-noise ratio, still avoiding saturation of the Brillouin amplifier. The output power was precisely adjusted before entering into the gas cell using a VOA in order to avoid any risk of saturation of the atomic transition [10]. The signal amplitudes after propagating through the fiber gas cell were recorded for different pump powers on a digital oscilloscope while the laser frequency was swept. 
(a)

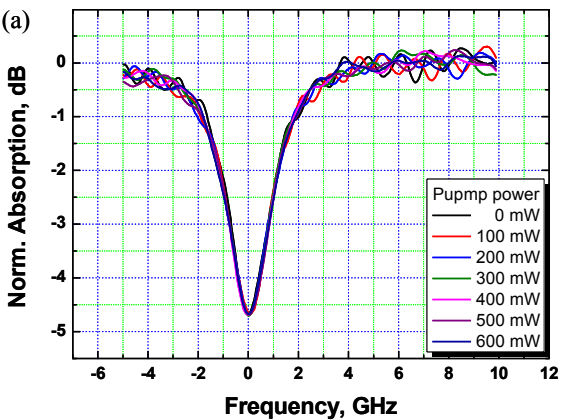

(b)

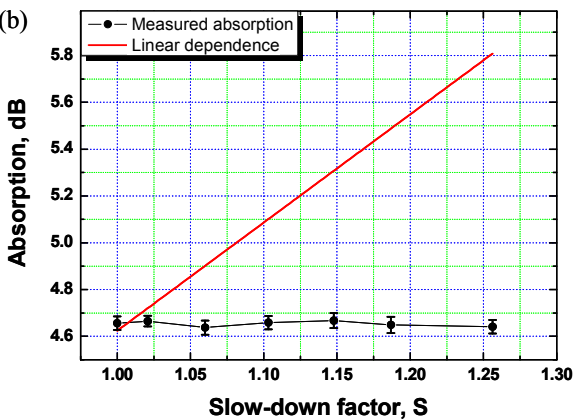

Figure.2: (a) Variation of the signal amplitude after propagating through the PCF gas cell in logarithmic scale for different pump powers. (b) Measured optical power loss at the peak attenuation due to the Beer-Lambert absorption as a function of the slow-down factor. The error bars show the measured standard deviation on the attenuation measurement and the red line represents the hypothetical response expected for an absorption coefficient inversely proportional to the group velocity.

Figure $2 \mathrm{a}$ shows the measured normalized absorption when the signal is centered in the $\mathrm{P}(17)$ absorption line at $1535.453 \mathrm{~nm}$. The pump power was incremented following the same sequence as during the group delay calibration. Therefore, the curves are fully representative of the gas absorption while the signal propagates through the gas cell at different group velocities. The absorption curves were normalized in logarithmic scale for a better comparison of the absorption levels. To evaluate the confidence level, measurements were repeated 5 times for each power levels, so that the mean value and the standard deviation of the peak attenuation due to the BL absorption could be accurately estimated. These values representing the absorption at the center of the molecular line are plotted as a function of the slow down factor in Figure 2b. It is clearly observed that BL absorption is totally independent on the slow-down factor $S$, hence the group velocity. The same experiment was also performed for the $\mathrm{P}(15)$ and $\mathrm{P}(19)$ absorption lines at $1534 \mathrm{~nm}$ and $1536 \mathrm{~nm}$, respectively, resulting in very similar results despite fairly different magnitudes for their absorption coefficient $\alpha$. The result of this experimental verification of the relationship between absorption and group velocity could be at first glance anticipated, since the BL absorption law is obtained from light-matter interaction by only considering the phase velocity.

\section{Conclusions}

We have experimentally clarified that the linear interaction between light and matter is not influenced by group velocity changes induced by modification of the material properties. The propagating velocity of a sinusoidally modulated light, which travels through a gas cell made of a single-mode PCF, was reduced using Brillouin slow light. The transmittance was then measured for different slow-down factors, resulting in no enhancement of gas absorption by slow light. It could be argued that the slow light induced delay $(\sim 10 \mathrm{~ns})$ is negligible compared to the signal period $(10 \mu \mathrm{s})$ of the sine wave used for the measurements and thus does not expand substantially the interaction time to lead to an observable effect. To decisively clarify this issue, we repeated this experiment using a 22 ns FWHM optical pulse as signal wave, shorter than the 45 ns propagation time through the gas fiber cell. This way the interaction time of the light pulse with the gas is clearly and noticeably modified by slow light. This experiment resulted in the same flat response, confirming that BL absorption is not influenced by group velocity.

We acknowledge the support from the Swiss National Science Foundation through project 200020_121860, the Spanish Ministry of Education and Science through project TEC2006-09990-C02-02 and the European Community's Seventh Framework Programme [FP7/2007-2013] under grant agreement n 219299 (GOSPEL project). This study was realized in the framework of the European COST Action 299 "FIDES".

\section{References}

[1] J. B. Khurgin and R. S. Tucker, Slow light: Science and applications (CRC Press, Boca Raton, 2009).

[2] M. Soljacic, S. G. Johnson, S. H. Fan, M. Ibanescu, E. Ippen and J. D. Joannopoulos, "Photonic-crystal slow-light enhancement of nonlinear phase sensitivity," J. Opt. Soc. Am. B, 19, 2052-2059 (2002).

[3] C. Mohat, B. Corcoran, M. E. Heidari, C. Grillet, B. J. Eggleton, T. P. White, L. O'Faolain and T. F. Krauss, "Slow light enhancement of nonlinear effects in silicon engineered photonic crystal waveguides," Opt. Express, 17, 2944-2953 (2008).

[4] A. L. Gaeta, "Slow light: Putting the brakes on images," Nature Photonics, 1, 140-141 (2007).

[5] Z. Shi, R. W. Boyd, D. J. Gauthier and C. C. Dudley, "Enhancing the spectral sensitivity of interferometers using slow-light media," Opt. Lett. 32, 915-917 (2007).

[6] M. Terrel, M. J. F. Digonnet and S. Fan, "Performance comparison of slow-light coupled-resonator optical gyroscopes," Laser \& Photon. Rev. 1-14 (2009).

[7] N. A. Mortensen and S. Xiao, "Slow-light enhancement of Beer-Lambert-Bouguer absorption," Appl. Phys. Lett. 90, 141108 (2007).

[8] K. H. Jensen et al, "Slow-light enhanced light-matter interactions with applications to gas sensing, " Opt. Commun. 281, 5335-5339 (2008).

[9] A. Hochman and Y. Leviatan, "Efficient and spurious-free integral-equation-based optical waveguide mode solver," Opt. Express, 15, 14431-14453 (2007).

[10] J. Henningsen, J. Hal and J. C. Petersen, "Saturated absorption in acetylene and hydrogen cyanide in hollow-core photonic bandgap fibers," Opt. Express, 13, 10475-10482 (2006). 